\title{
Role of the perfluoro effect in the selective photochemical isomerization of hexafluorobenzene
}

\author{
Jordan M. Cox ${ }^{1 \ddagger}$, Mike Kellogg ${ }^{2 \ddagger}$, Matthew Bain ${ }^{2}$, Stephen Bradforth*2, Steven A. Lopez*1 \\ ${ }^{I}$ Department of Chemistry and Chemical Biology, Northeastern University, Boston, MA, USA \\ ${ }^{2}$ Department of Chemistry, University of Southern California, Los Angeles, CA 90089-0482, USA.
}

Corresponding author e-mail: stephen.bradforth@usc.edu, s.lopez@,northeastern.edu

*These authors contributed equally to the manuscript.

\begin{abstract}
Hexafluorobenzene and many of its derivatives exhibit a chemoselective photochemical isomerization, resulting in highly-strained, Dewar-type bicyclohexenes. While the changes in absorption and emission associated with benzene hexafluorination have been attributed to the socalled "perfluoro effect," the resulting electronic structure and photochemical reactivity of hexafluorobenzene are still unclear. We now use a combination of ultrafast time-resolved spectroscopy, multiconfigurational computations, and non-adiabatic dynamics simulations to develop a holistic description of the absorption, emission, and photochemical dynamics of the $4 \pi$ electrocyclic ring-closing of hexafluorobenzene and the fluorination effect along the reaction coordinate. Our calculations suggest that the electron-withdrawing fluorine substituents induce a vibronic coupling between the lowest-energy ${ }^{1} B_{2 u}\left(\pi \pi^{*}\right)$ and ${ }^{1} E_{l g}\left(\pi \sigma^{*}\right)$ excited states by selectively stabilizing the $\sigma$-type states. The vibronic coupling occurs along vibrational modes of $e_{2 u}$ symmetry which distorts the excited-state minimum geometry resulting in the experimentally broad, featureless absorption bands, and a $\sim 100 \mathrm{~nm}$ Stokes shift in fluorescencein stark contrast to benzene. Finally, the vibronic coupling is shown to simultaneously destabilize the reaction pathway towards hexafluoro-benzvalene and promote molecular vibrations along the $4 \pi$ ring-closing pathway, resulting in the chemoselectivity for hexafluoro-Dewar-benzene.
\end{abstract}

\section{Introduction}

The synthesis of polyacetylene sparked the field of organic electronics, despite difficulties in processing polyacetylene and its high propensity to be oxidized when exposed to air. ${ }^{1,2}$ As early as 1979 , polymer chemists have sought to circumvent these challenges, especially its instability, with a strategy of fluorination. Theoretical calculations predict that the electron-withdrawing fluorines significantly reduces the oxidation rate by lowering the frontier molecular orbitals of polyacetylene and minimizing overlap with those of molecular oxygen. However, synthesizing fluorinated polyacetylenes has proved difficult by the traditional means of polymerizing fluorinated acetylene. ${ }^{3}$ Not only is difluoroacetylene an explosive and pyrophoric gas, but polymerization results in a highly irregular material that contains a mixture of $-\mathrm{CF},-\mathrm{CF}_{2}$, and $\mathrm{CF}_{3}$ functional groups. Burns and co-workers ${ }^{4}$ recently achieved this elusive synthesis by leveraging the mechanochemical "unzipping" of highly strained ladderene polymers to form partially fluorinated polyacetylenes. The key step in their synthetic scheme is the formation of a fluorinated ladderene, achieved through a photochemical cascade reaction, involving a 
hexafluorobenzene (HFB) [2+2]-cycloaddition, followed by a disrotatory $4 \pi$-electrocyclic ringclosing of the hexafluorinated diene intermediate (Figure 1b).
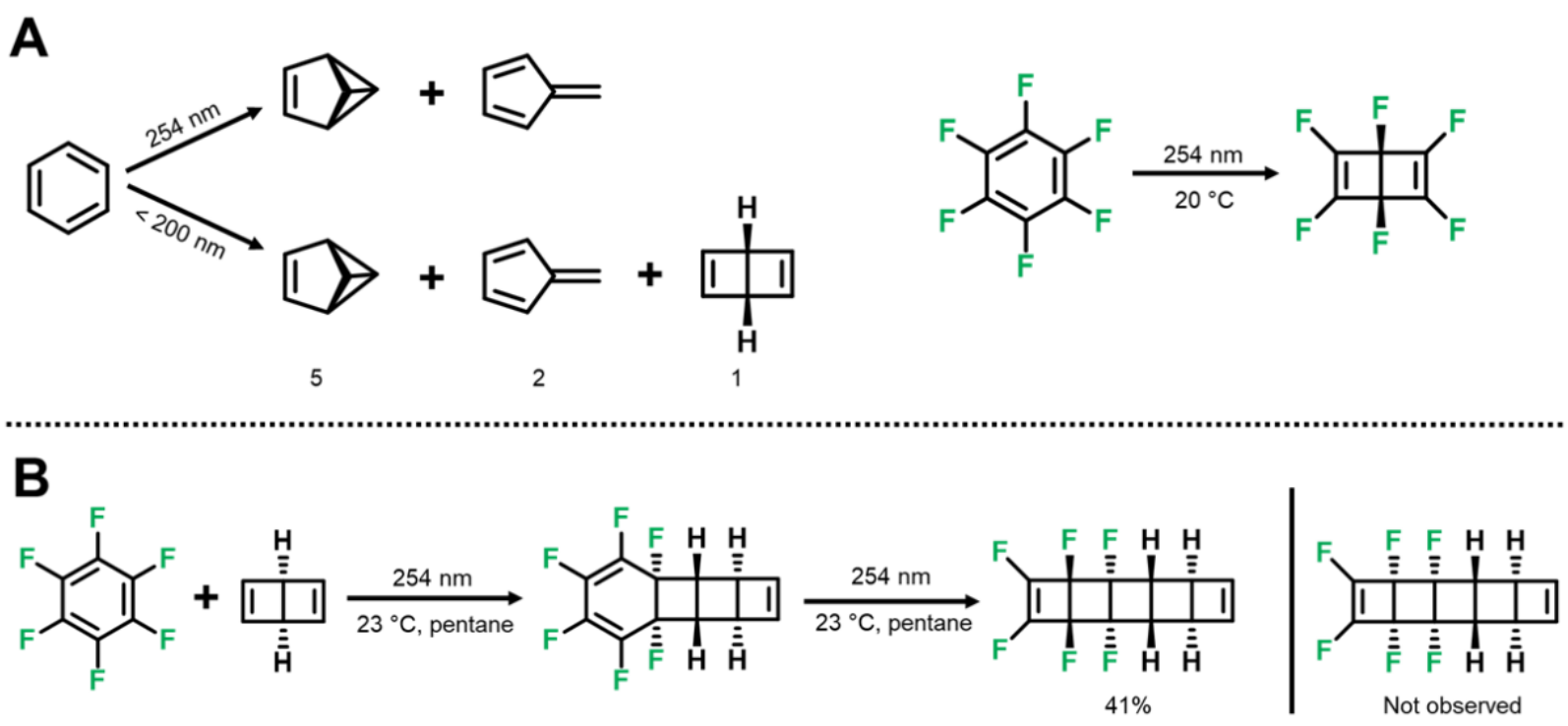

Figure 1. (A) Photochemical isomerization reactions of benzene and HFB yielding different product distributions. $^{5}$ (B) Photochemical cascade reaction of Burns and co-workers. ${ }^{4}$

The $4 \pi$ electrocyclic ring-closing reaction is known for many highly fluorinated cyclic polyenes, many of which result from cycloadditions with HFB. ${ }^{6-9}$ HFB itself undergoes the same ringclosing reaction, ${ }^{10}$ photochemically isomerizing to hexafluoro-Dewar-benzene (Dewar-HFB) chemoselectively. The hexafluoro-benzvalene or -fulvene isomers are not observed, but are the major products of benzene photochemistry (Figure 1a). ${ }^{11,12} \mathrm{HFB}$ also shows significantly different photophysical behavior than benzene; its absorption spectrum has a broad, featureless absorption band and a substantial $(100 \mathrm{~nm})$ Stokes shift in the emission band, even in the gas phase. ${ }^{13}$ These dramatic changes in photophysics have been attributed to the "perfluoro effect". ${ }^{14}$ The stark contrast in the photochemical outcomes of HFB and benzene photochemistries suggests that the perfluoro effect also plays an important role as HFB isomerizes to Dewar HFB.

The chemoselective isomerization of HFB was first reported in 1966 when Haller and Camaggi 10,15 independently reported the reaction. Further work by Haller determined that the quantum yield of the gas-phase isomerization was $\leq 3 \%$, under pressures ranging from 44 to 1052 torr. ${ }^{16}$ The quantum yield was unchanged in the presence of triplet- sensitizer, $\mathrm{O}_{2}$. This suggests that the reaction proceeds entirely in the singlet manifold. Picosecond transient absorption (TA) spectroscopy indicates the possibility of diradical or charge-transfer intermediates, though the role of these species in the photochemistry is still unknown. ${ }^{17}$ This quantum yield is low compared to other examples of $4 \pi$ electrocyclic ring-closing reactions where quantum yields can reach $17 \% .{ }^{18}$

Laser-induced fluorescence measurements by Zgierski et al. show that benzene perfluorination substantially lowers the energies of the $\mathrm{C}-\mathrm{F} \sigma$ and $\sigma^{*}$ orbitals. ${ }^{13}$ In fact, Zgierski proposes that in $\mathrm{HFB}$, the $\sigma$-type orbital stabilization is sufficient to reverse the $\pi \pi^{*}$ and $\pi \sigma^{*}$ state-ordering relative to benzene and that the $S_{1}$ state in HFB is of $\pi \sigma^{*}$ character. ${ }^{13}$ The $\pi \sigma^{*} S_{1}$ assignment has been used 
by Zgierski and Temps ${ }^{13,19}$ to explain the large Stokes shift by assigning the gap between the absorption and emission onsets to an optically dark $S_{0} \rightarrow S_{1}$ transition of $\pi \sigma^{*}$ character. This idea has been used to explain the low fluorescence yield and short fluorescence lifetime of HFB. Philis et al., Motch et al., and Holland et al. instead agree that the $S_{1}$ and $S_{2}$ states are $\pi \pi^{*}$ and $\pi \sigma^{*}$, respectively based on photoabsorption and photoelectron spectroscopy measurements. ${ }^{20-22}$

Mondal et al. pioneered theoretical work on HFB dynamics, describing the initial ultrafast $\mathrm{S}_{\mathrm{N}} \rightarrow \mathrm{S}_{1}$ relaxation processes, and used equation-of-motion coupled-cluster (EOM-CCSD) calculations to support the $\mathrm{S}_{1}$ assignment of Philis, Motch, and Holland. ${ }^{23}$ However, a model of the photophysics and photochemistry of HFB which unifies the current reported observations does not yet exist. Developing this unified description would lay the foundation for design rules of highly strained compounds that leverage the chemoselective photoisomerization of HFB and its derivatives. This work utilizes ultrafast time-resolved spectroscopic studies, multireference calculations, and nonadiabatic molecular dynamics simulations to clarify the photophysics and origin of the low yielding, chemoselective isomerization to Dewar-HFB. While this photochemical reaction is synthetically useful, ${ }^{4}$ this work shows why this photochemical reaction is inefficient and emphasizes the difficulty in tracking such reaction pathways.

\section{Results \& Discussion}

The density of frontier excited states and the highly symmetric $D_{6 h}$ structure of HFB has generated substantial debate in the literature about the nature of the light-induced electronic transitions. The $\pi \pi^{*}$ or $\pi \sigma^{*}$ character of these states dictates the shape of the potential energy surface which directs the photochemistry. Without a full understanding of the order and interplay between the molecular excited states of HFB, a clear understanding of the inefficient yet chemoselective reactivity has remained elusive.

Figure 2 shows the overlay of experimental and simulated absorption spectra for HFB, with the contributions of each of the four lowest-energy electronic states to the simulated spectrum. The steady-state solution-phase UV-Vis absorption spectrum, shown by the dashed red trace, was measured for a $75 \mu \mathrm{M}$ solution of HFB in ethanol. The molar absorptivities in the measured transitions lie in the $200-600 \mathrm{M}^{-1} \mathrm{~cm}^{-1}$ range, indicating that HFB is a relatively weak absorber for these lowest energy transitions - an intuitively undesirable quality in a photochemical reagent.

The simulated absorption spectrum, the solid black trace, was computed at the extended multistate (XMS-)CASPT2(6,7)/aug-cc-pVDZ level of theory based on 150 HFB geometries randomly sampled from a $1 \mathrm{ps}$ SA-10-CAS(6,7)/6-31G molecular dynamics trajectory propagated in the ground state at $300 \mathrm{~K}$ in vacuum. The absorption line-spectrum computed at the XMSCASPT2 $(6,7) /$ aug-cc-pVDZ level for the $D_{6 h}$-symmetric minimum geometry predicts absorption oscillator strengths of $1.7 \times 10^{-5}$ and $7.6 \times 10^{-5}$ for the ${ }^{1} B_{2 u}$ and ${ }^{1} B_{1 u}\left(\pi \pi^{*}\right)$ states, respectively, and $1.9 \times 10^{-9}$ for the ${ }^{1} E_{l g}\left(\pi \sigma^{*}\right)$ state. The computed absorption spectrum in Figure 2 encompasses geometries that break the $D_{6} \mathrm{~h}$ symmetry leading to a much higher average oscillator strength of $6.7 \times 10^{-3}$ and $2.4 \times 10^{-3}$ for the two ${ }^{1} \mathrm{E}_{1 \mathrm{~g}}$ states, which is comparable to the ${ }^{1} \mathrm{~B}_{2 \mathrm{u}}$ and ${ }^{1} \mathrm{~B}_{1 \mathrm{u}}$ states at $3.2 \times 10^{-3}$ and $9.0 \times 10^{-2}$, respectively. 


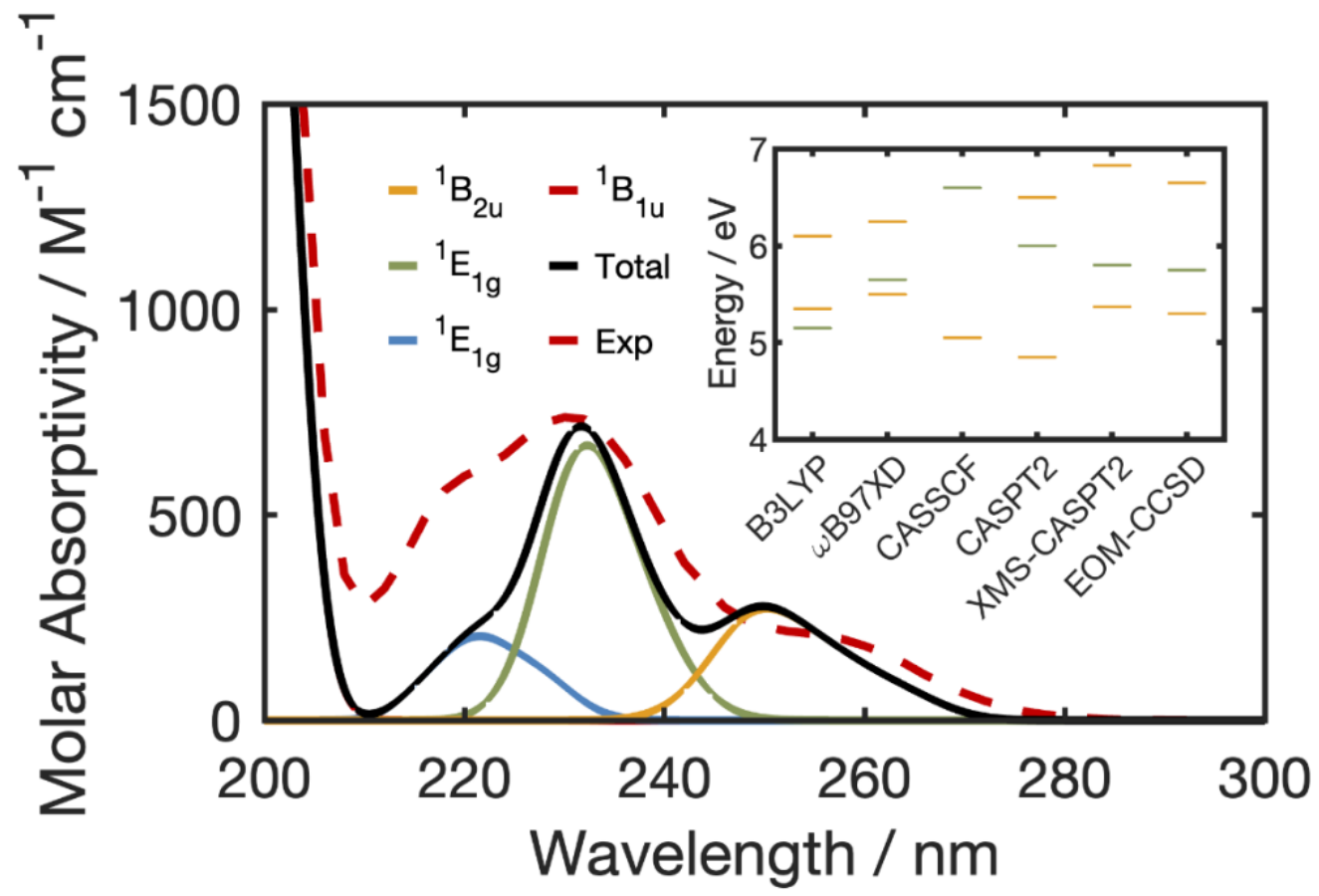

Figure 2. The theoretically computed absorption of HFB in black and the measured UV-Vis absorption spectrum of HFB solvated in ethanol in dashed red. The transition-specific computed spectra are rendered below in solid-colored lines with upper state symmetry assignments shown in the legend. The inset panel shows the vertical excitation energies for the three lowest excited states of HFB. All calculations used the aug-cc-pVDZ basis set, CAS and (XMS-CASPT2) calculations included a $(6,7)$ active space described in the experimental section. The orange traces indicate $\pi \pi^{*}$ character while the green traces indicate $\pi \sigma^{*}$ character.

The colored curves in Figure 2 show the contributions to the total absorption spectrum computed for each of the three lowest-energy electronically excited states. The calculated absorption spectrum suggests that the lowest-energy excited state is the ${ }^{1} B 2 u\left(\pi \pi^{*}\right)$ state and that the absorption peaks at 230 and $220 \mathrm{~nm}$ arise from splitting of the ${ }^{1} E_{1 g}\left(\pi \sigma^{*}\right)$ state. This assignment matches the previous experimental work of Philis, Motch, and Holland and the theoretical work of Mondal. Mondal demonstrated that the splitting of the ${ }^{l} E_{l g}$ state resulted from the Jahn-Teller effect but disagrees with Temps and co-workers who assign the ${ }^{l} E_{1 g}$ state as $\mathrm{S}_{1}$. The Temps assignment is bolstered by providing an intuitive explanation of the large gap between the absorption and emission onsets of $100 \mathrm{~nm}$ by suggesting that this gap arises instead from emission from a lowlying ${ }^{l} E_{\lg }\left(\pi \sigma^{*}\right)$ dark state centered at around $280 \mathrm{~nm}$. Therefore, for the assignment of $\mathrm{S}_{1}$ as $\pi \pi^{*}$ to be correct, an unusually large Stokes shift would require an $S_{1}$ potential energy surface where the excited state minimum geometry is significantly different from the ground state minimum $-\mathrm{a}$ potentially important observation in understanding the photochemistry.

To determine the origins of the $100 \mathrm{~nm}$ Stokes shift observed in the absorption/fluorescence spectra of HFB, we present the absorption and fluorescence emission spectra of a $75 \mu \mathrm{M}$ solution of HFB in ethanol with a solid red line (absorption) and red filled trace (fluorescence) in the main panel of Figure 3. 


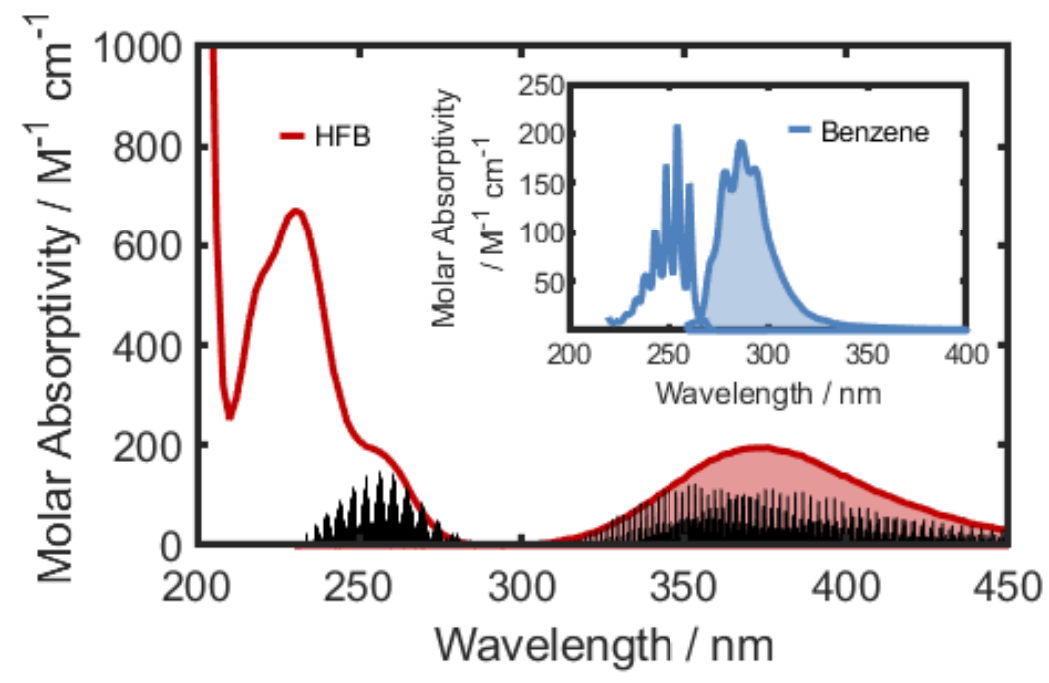

Figure 3. Absorption and emission spectra for hexafluorobenzene (red, main panel) and benzene (blue, inset panel). Absorption spectra are rendered in solid lines and emission spectra in filled traces. Both absorption and emission spectra for hexafluorobenzene are measured using a $75 \mu \mathrm{M}$ solution in ethanol, while the analogous benzene spectra are adapted from $\mathrm{Du}$ et. $a{ }^{24}$ and the extinction values calibrated by Berlman. ${ }^{25}$ The black stick spectra represent vibrationally resolved absorption and emission spectra computed from Franck-Condon factors of the ground- and ${ }^{1} B_{2 u}$ excited states. Franck-Condon factors are computed using the parallel mode approximation at $0 \mathrm{~K}$.

Beneath these experimental traces is a black stick spectrum showing the computed vibrationallyresolved absorption and emission spectra between the ${ }^{1} \mathrm{~A} 1 \mathrm{~g}$ and ${ }^{1} B_{2 u}\left(\pi \pi^{*}\right)$ states of the isolated molecule. The inset panel shows the equivalent spectra for benzene in blue, where the absorption and fluorescence were adapted from $\mathrm{Du}$ et al. ${ }^{24}$ and the absorption scaled by the Berlman extinction coefficient. ${ }^{25}$ The vibrationally resolved electronic spectra were simulated by computing the Franck-Condon factors for the overlap between the ${ }^{1} A 1 g$ and ${ }^{l} B_{2 u}$ state vibrational wavefunctions using the harmonic approximation as implemented in ezSpectrum. ${ }^{26}$ These computed spectra consider the 14 most significant vibrational modes and include up to 21 and 30 vibrational quanta in the absorption and emission spectra, respectively.

Figure 3 shows the overlay of the experimental and simulated vibrationally resolved electronic spectra. The full set of vibrational modes and their corresponding frequencies are shown in Table S1. The Franck-Condon overlap between the ${ }^{l} A_{1 g}$ and ${ }^{l} B_{2 u}$ states suffices to explain the origin of the large Stokes shift of $1.3 \mathrm{eV}(260-360 \mathrm{~nm})$ between the absorption and emission spectra. This computed value slightly underestimates the experimentally measured Stokes shift of $1.56 \mathrm{eV}$. This conclusively demonstrates that the Stokes shift is an inherent feature of the ${ }^{1} B_{2 u}$ state, rather than an artifact from a dark emissive state.

The large value of the Stokes shift is equivalent to recognizing there is a large displacement along one or more vibrational modes. Our analysis of the vibrationally resolved electronic spectra, which shows the most intense absorption and emission features deposit 10 and 14 vibrational quanta in 
the excited-state and ground-state, respectively. These vibrational quanta are distributed through several combination progressions and predominantly activate the $v_{18}$ and $v_{19}$ modes, as rendered in Table S1 and using the notation of Herzberg. ${ }^{27}$ These modes have $e_{2 u}$ symmetry in $D_{6 h}$ and correspond to out-of-plane bending of the para-oriented carbon and fluorine atoms, respectively. The selective population of the $e_{2 u} u$ vibrational modes suggests a symmetry-dependent coupling dominates the absorption and emission spectra.

\section{Electronic State Coupling}

To establish an intuitive understanding of the origin of the Stokes shift and vibrationally resolved electronic spectra, the electronic and minimum geometry structures of the ground and ${ }^{1} B_{2 u}$ states and the motions connecting them are examined. The left-hand side of Figure 4 shows the completely planar $D_{6 h}$ geometry of the ground state minimum and the minimum-energy geometry of the ${ }^{1} B_{2 u}$ state. The excited state minimum is significantly distorted compared to the highsymmetry ground state geometry. Two of the carbon atoms, with a 1,4 relationship, are visibly distorted out-of-plane, and the fluorine atoms attached to the 1,4-carbons are bent out-of-plane by $18^{\circ}$. This results in a $C_{2 v}$ symmetric geometry. This is in contrast to calculations of the optimized excited state minima of benzene, where the ${ }^{1} B_{2 u}$ minimum-energy geometry is $D_{6} h$ symmetric. ${ }^{28}$ This significant geometric distortion from $\mathrm{S}_{0}$ to $\mathrm{S}_{1}$ results in the observed Stokes shift by reducing the $\mathrm{S}_{1} / \mathrm{S}_{0}$ gap by $1.53 \mathrm{eV}$.

The minimum energy path (MEP) from the Franck-Condon point on the ${ }^{1} B_{2 u}$ surface is computed to investigate the evolution of the electronic structure of HFB throughout this geometric distortion. There is a sudden shift in the multiconfigurational nature of the CAS wavefunction along the MEP upon breaking the $D_{6 h}$ symmetry (see Figure S1). The wavefunction is entirely comprised of $\pi \pi^{*}$ configurations while the molecule is $D_{6 h}$ symmetric; symmetry breaking along the MEP induces $\pi \sigma^{*}$ mixing. The maximum $\pi \sigma^{*}$ contribution to the total wavefunction is $6.6 \%$. Taken together, the $\pi \pi^{*}$ and $\pi \sigma^{*}$ configuration mixing with the symmetry-breaking distortion suggest that the ${ }^{1} B_{2 u}$ state is vibronically coupled with the energetically nearby ${ }^{1} E_{\lg }\left(\pi \sigma^{*}\right)$ state.

As this coupling should occur via $e 2 u$ vibrational modes, an energy plot of the ground-state and the first four excited-states with respect to $e_{2 u}$ normal mode displacement is shown in the right of Figure 4 . The orange trace shows the double-well stabilization of the ${ }^{1} B_{2 u}$ state when displaced along the $e_{2 u}$ symmetric modes leading to the distorted minimum energy geometry. The blue and green traces represent the splitting of the two components of the ${ }^{1} E_{1 g}$ state. Previous work has shown that the vibronic coupling constant between the ${ }^{1} B_{2 u}$ and ${ }^{1} E_{1 g}$ states is 0.1548 and 0.0619 $\mathrm{eV}$ for coupling through modes $v_{18}$ and $v_{19}$, respectively. ${ }^{23}$ The cross-section of the potential energy surface (PES) in Figure 4 shows that the combination of the vibronic coupling constant and the low energy gap is sufficient to induce the instability of the $D_{6 h}$ geometry of the HFB ${ }^{1} B_{2 u}$ state with respect to distortions along the $e_{2 u}$ modes. We conclude that the small energy gap $(0.42 \mathrm{eV})$ between the ${ }^{1} B_{2 u}$ and ${ }^{1} E_{1 g}$ states in the $\mathrm{FC}$ region results from the perfluoro effect, implying that Stokes shift, in turn, results from the perfluoro effect via a pseudo-Jahn-Teller distortion in the ${ }^{1} B_{2 u}\left(\pi \pi^{*}\right)$ state. Further motion along this $e_{2 u}$ mode from the ${ }^{1} B_{2 u}$ minimum geometry leads to Dewar-HFB, implying that this coupling encourages Dewar-HFB formation. 

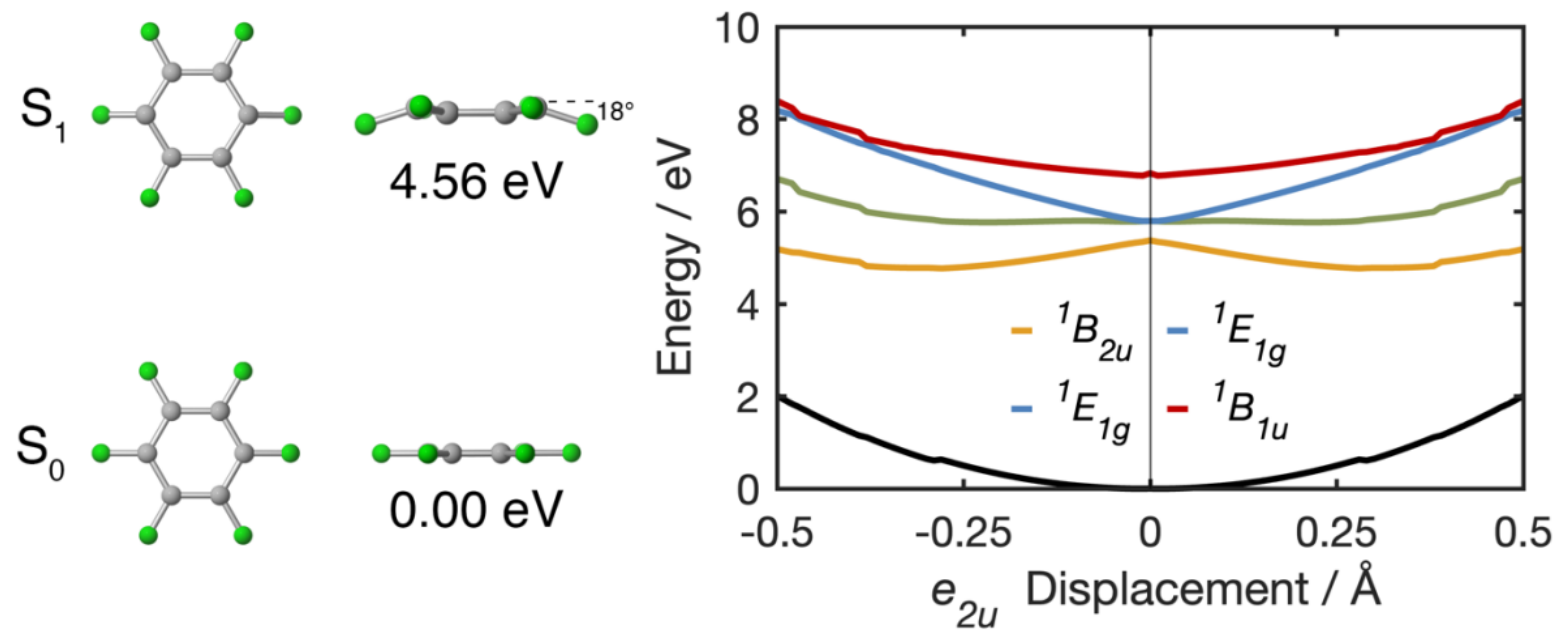

Figure 4. (Left) side and top elevations of the minimum energy geometries for the $\mathrm{S}_{0}\left({ }^{1} \mathrm{~A}_{1 \mathrm{~g}}\right)$ and $\mathrm{S}_{1}\left({ }^{1} \mathrm{~B}_{2 \mathrm{u}}\right)$ states. (Right) Cuts through the ground, ${ }^{1} \mathrm{~A}_{1 \mathrm{~g}}$ (black), and first four excited, ${ }^{1} \mathrm{~B}_{2 \mathrm{u}}$ (orange), ${ }^{1} \mathrm{E}_{1 \mathrm{~g}}$ (green), ${ }^{1} \mathrm{E}_{1 \mathrm{~g}}$ (green), and ${ }^{1} \mathrm{~B}_{1 \mathrm{u}}$ (red), potential energy surfaces, along the $v_{18} \mathrm{e}_{2 \mathrm{u}}$ motion corresponding to opposing carbons moving symmetrically out of plane.

\section{Transient Absorption Spectroscopy}

With the multiconfigurational calculations reproducing the experimental photophysical processes in HFB, the photochemical isomerization of HFB to Dewar-HFB is now considered. Figure 5 shows the experimental transient absorption spectrum of a $200 \mathrm{mM}$ solution of HFB in ethanol recorded following excitation with a $255 \mathrm{~nm}$ UV laser pulse $(580 \mathrm{~nJ})$. This wavelength was selected to match the $255 \mathrm{~nm}$ lamps employed in gram scale reactors for these syntheses. ${ }^{4}$ The transient absorption spectrum is rendered at a series of pump-probe delays denoted by colorcoordinated legend. The stick spectrum represents the computed excited state absorption spectra at the $\mathrm{S}_{1}$ minimum energy geometry. Each absorption peak in the stick spectrum was convoluted with a Gaussian function with an (arbitrary) full-width half-max of $0.2 \mathrm{eV}$, which yielded the simulated absorption spectrum shown by the red trace. The corresponding contour plot and the full range of pump-probe delays are rendered in Figure S2 and Table S2.

By analyzing the ground state absorption spectrum (Figure 2), approximately $3 \%$ of the molecules excited at $255 \mathrm{~nm}$ are estimated to be promoted to the $\mathrm{S}_{2}{ }^{1} E_{1 g}$ state and $97 \%$ promoted to the $\mathrm{S}_{1}$ ${ }^{1} B_{2 u}$ state. The transient spectrum contains two excited-state absorption features longer than 400 $\mathrm{nm}$, overlapping a (negative) stimulated emission band peaking at $375 \mathrm{~nm}$ (compare to fluorescence band in Figure 3). The excited-state absorption from $\mathrm{S}_{1}$ features a broad absorption peak centered at $550 \mathrm{~nm}$ that immediately rises and a sharper, more intense absorption centered at $440 \mathrm{~nm}$, which rises to $90 \%$ of its maximum intensity instantaneously and rises to $100 \%$ over $\sim 10$ ps.

A broad $550 \mathrm{~nm}$ absorption has previously been assigned to a biradical form of HFB based upon then-current $\mathrm{CNDO} / \mathrm{S}$ calculations. ${ }^{17} \mathrm{We}$ prefer the assignment to an excited state $\mathrm{S}_{1}$ because stimulated emission is simultaneously observed. Our multiconfigurational calculations predict an 
excited-state spectrum based on the $\mathrm{S}_{1}$ minimum geometry. This spectrum shows two dominant absorption peaks at $540 \mathrm{~nm}$ and $590 \mathrm{~nm}$. These peaks correspond, in $\mathrm{C}_{2} \mathrm{~V}$ symmetry, to the $\mathrm{S}_{1}\left({ }^{1} \mathrm{~B}_{1}\right)$ $\rightarrow \mathrm{S}_{3}\left({ }^{1} \mathrm{~B}_{1}\right)$ and $\mathrm{S}_{1}\left({ }^{1} \mathrm{~B}_{1}\right) \rightarrow \mathrm{S}_{4}\left({ }^{1} \mathrm{~A}_{1}\right)$ transitions, respectively. These two transitions from the $\mathrm{S}_{1}$ minimum overlap to form one peak and better explain the broad absorption feature at $550 \mathrm{~nm}$ in the experimental spectrum. Based on the conditions in our experiment, we calculate $\sim 0.04 \%$ of HFB molecules are photoexcited. The XMS-CASPT2/aug-cc-pVDZ calculations predict oscillator strengths of 0.0014 and 0.0057 for the $\mathrm{S}_{1}\left({ }^{1} \mathrm{~B}_{1}\right) \rightarrow \mathrm{S}_{3}\left({ }^{1} \mathrm{~B}_{1}\right)$ and $\mathrm{S}_{1}\left({ }^{1} \mathrm{~B}_{1}\right) \rightarrow \mathrm{S}_{4}\left({ }^{1} \mathrm{~A}_{1}\right)$, respectively. From these values, and the estimated concentration of excited-state molecules, an excited state molar absorptivity of 500, and in turn, an excited state optical density of $0.5 \mathrm{mOD}$ are predicted; both values agree well with the recorded transient absorption measurement. This calculation follows Equation S1. However, an assignment for the $440 \mathrm{~nm}$ peak is more difficult as it is completely absent from the computed spectrum.

Turning to the $440 \mathrm{~nm}$ spectral feature, a rising absorption could be assigned to a new absorbing species formed by a bimolecular process. (Figure S3 shows that the sharpening of the $440 \mathrm{~nm}$ feature cannot be explained simply as a weakening subtractive effect from the nearby stimulated emission peak narrowing following the cooling of the solvent environment) A rising band centered at this same wavelength has previously been assigned by Varma et al. to both cation and anion absorption bands using CNDO/S calculations. ${ }^{17}$ Varma thus speculated that charge transfer occurs between HFB pairs during the 10 ps rise-time, consistent with the diffusion timescale of charge transfer partners finding one another. EOM-IP-CCSD/aug-cc-pVDZ and EOM-EA-CCSD/aug-ccpVDZ calculations of the isolated cation and anion absorption bands, respectively, predict that within the $350-650 \mathrm{~nm}$ spectral range of the TA experiment, the cation has a single strong absorption centered at $385 \mathrm{~nm}(3.23 \mathrm{eV})$ with an oscillator strength of 0.13 . In contrast, the anion has three strong absorption bands at $483 \mathrm{~nm}(2.57 \mathrm{eV}), 445 \mathrm{~nm}(2.78 \mathrm{eV})$, and $368 \mathrm{~nm}(3.37 \mathrm{eV})$ with oscillator strengths of 0.190 .14 and 0.077 , respectively. The computed bands and their oscillator strengths are summarized in Table S3. These oscillator strengths are $\sim 10^{4}$ stronger than the excited state absorption bands. If correct, they must belie a correspondingly lower concentration of charge transfer contact pairs to yield optical densities on the same order as the $\mathrm{S}_{1}$ excited state absorption. The anion band predicted at $445 \mathrm{~nm}$ provides a convenient explanation for the $440 \mathrm{~nm}$ band in the TA spectrum. However, the conspicuous lack of a corresponding cation peak at $386 \mathrm{~nm}$ - which would also need to show the same 10 ps rise time - brings this assignment into question. Moreover, transient absorption data recorded in non-polar cyclohexane, shown in Figures S4 and S5, shows no apparent diminishment or shifting of the $440 \mathrm{~nm}$ band, as one would when a polar environment no longer stabilizes charged species.

An alternate explanation for a time-delayed $440 \mathrm{~nm}$ absorption band might therefore be excimer formation. ${ }^{29}$ Such rearrangement from a ground-state T-shape to an excited-state slip-stacked arrangement of two aromatic rings occurs on a 10 - 18 ps timescale in pre-formed dispersionbound dimers of benzene in the gas phase. ${ }^{30}$ However, in dilute solutions, like those studied here, the dimer formation timescale should be longer due to the additional diffusion time necessary to bring the rings together. Moreover, the formation time should scale with HFB concentration; and the cyclohexane data in Figure S4/S5 rises on a comparable timescale despite HFB being 4 times more dilute (Figure S10). The assignment of this peak, therefore, remains somewhat elusive. 
The inset panel in Figure 5 shows the spectral intensity at two wavelengths, $440 \mathrm{~nm}$ (blue) and 550 $\mathrm{nm}$ (red), as a function of pump-probe time delay. The time axis is split with the -1 to $1 \mathrm{ps}$ region rendered linearly, while the 1 to $1000 \mathrm{ps}$ region is plotted logarithmically in time. The majority of the excited-state signal is formed within the experimental IRF and persists for a lifetime significantly greater than the window of the experiment $(1 \mathrm{~ns})$.

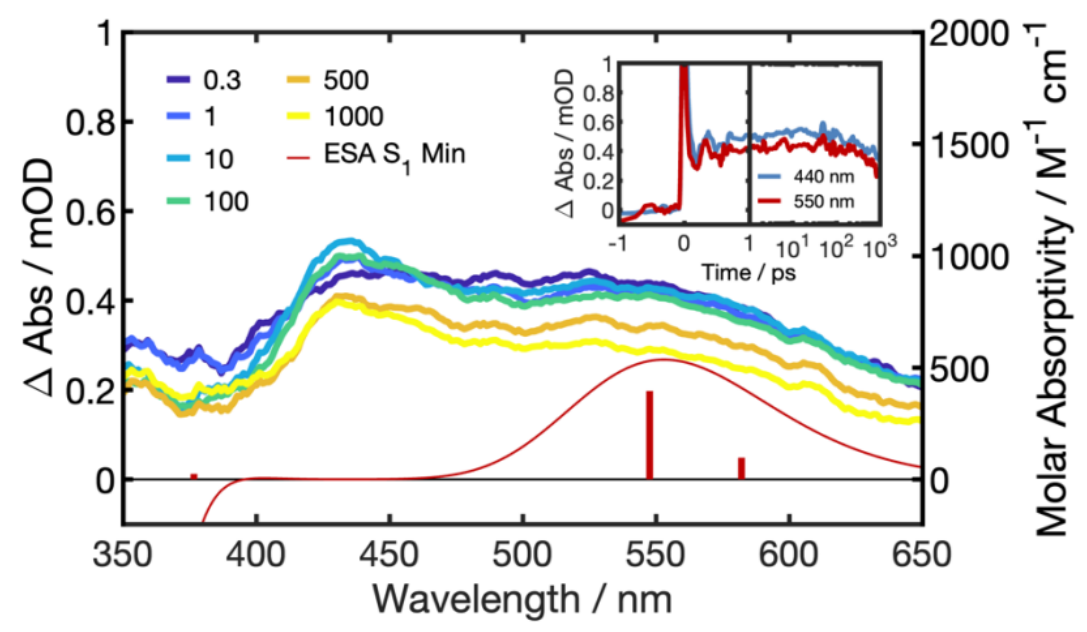

Figure 5. Transient absorption spectra for $200 \mathrm{mM}$ HFB in ethanol. The main panel shows transient absorption spectra at pump-probe delays indicated by the color-coded legend (in picoseconds) following photoexcitation at $255 \mathrm{~nm}(4.86 \mathrm{eV})$. The inset shows the time evolution on a linear scale from -1 to $1 \mathrm{ps}$ pump-probe delay and a logarithmic scale from 1 to $1000 \mathrm{ps}$ delays. For sticks and simulated bands see the text.

Notice that rapidly damped oscillations show up at both probe wavelengths; these oscillations in the ESA intensity over the first picosecond are fit to a convolution of a damped sine wave and an exponential decay. A rendering of this fit and the resultant constants can be found in Figure S6 and Table S4, respectively, along with the model's fully functional form. The oscillation frequency is $\sim 110 \mathrm{~cm}^{-1}$, which most closely matches the lowest frequency normal mode $v_{19}$ (computed as 157 $\mathrm{cm}^{-1}$ in the ground state) - one of the two vibrational modes implicated in the vibrationally resolved absorption to the ${ }^{1} B_{2 u}$ state. $93 \mathrm{~cm}^{-1}$ oscillations with a longer damping time ( $\sim 1 \mathrm{ps}$ compared to $\sim 360$ fs seen here) were also observed in the ion-detected pump-probe experiments of Temps for gas-phase HFB and assigned to the same motion. ${ }^{19}$

There is no signal in the TA spectra assignable to the Dewar-HFB photoproduct. We would expect Dewar-HFB product to absorb well to the blue of the probe window of the experiment. The maximum transient absorption signal of $0.5 \mathrm{mOD}$ is weak relative to typical molecules measured with this technique: this can be ascribed to both the weak excited state absorption strengths (1000 $\mathrm{M}^{-1} \mathrm{~cm}^{-1}$ ) possibly compounded low excited state population.

To cross check that a significant fraction of excited state population has not branched at earlier delays than captured in the current $\sim 50$ fs resolution study, we make a comparison to the excited state evolution of benzene. The excited state of benzene has no ultrafast deactivation pathways and the quantum yield of the photoproduct channel is $<1 \% .{ }^{31}$ Thus, picosecond-scale excited state 
signals in benzene should be attributable to the full excited state population. Using the same computational methods as applied to HFB, the calculated excited molar state absorptivity of benzene is determined to be $200 \mathrm{M}^{-1} \mathrm{~cm}^{-1}$. This is comparable with HFB at $500 \mathrm{M}^{-1} \mathrm{~cm}^{-1}$ allowing estimation of the HFB excited state population if the data is recorded under identical conditions. A comparison of the TA spectra of HFB (green) and benzene (blue) recorded back-toback in a single experimental run is shown in Figure 6. Both of these transient shown were recorded at a pump-probe delay time of $1 \mathrm{ps}$ to avoid interference either by solvent two-photon absorption at early times or benzene excimer formation at later times. The HFB and benzene solutions were prepared with identical ground state optical densities to ensure the same number of excited state molecules were formed. Very similar transient absorbances are measured. This indicates that, similar to benzene, the TA signal captures the majority of the HFB molecules excited, and rules out the possibility of a significant bifurcated flux back to the ground state, or to Dewar-HFB formation, at early times.

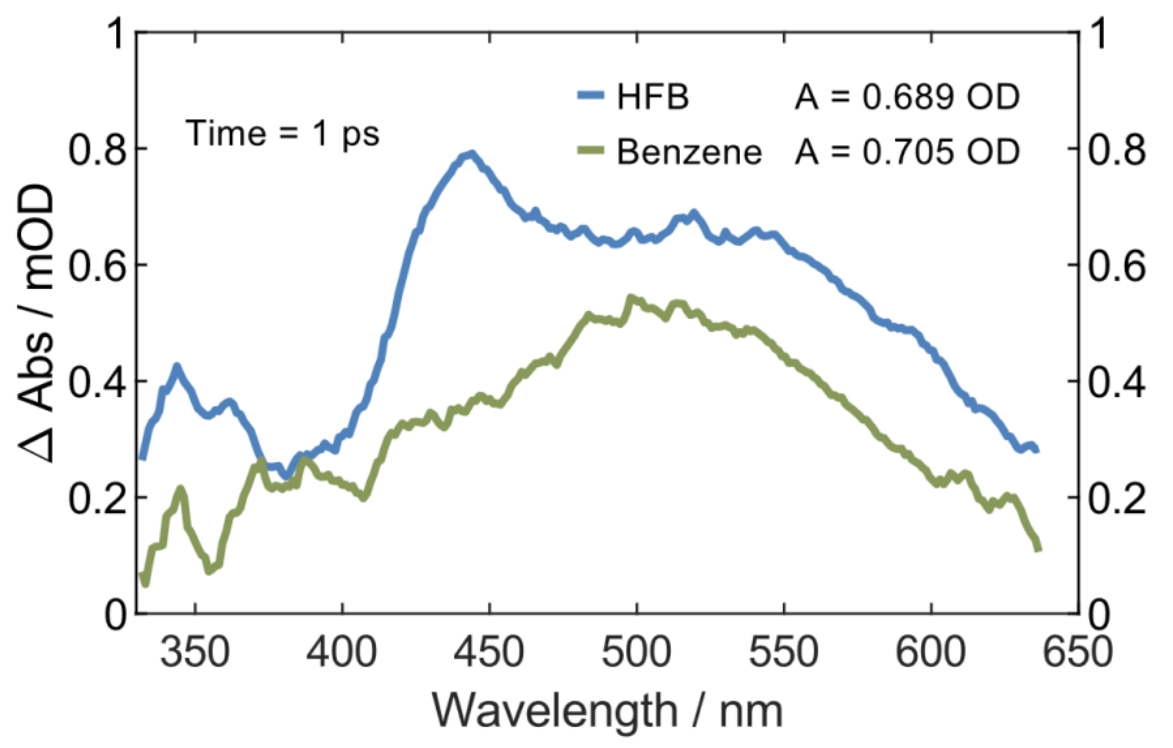

Figure 6. Transient absorption spectra of benzene (green) and HFB (blue) taken 1 ps after excitation with $266 \mathrm{~nm}$. Benzene's concentration was $\sim 4$ M and HFB was $400 \mathrm{mM}$. These spectra were captured at $1 \mathrm{ps}$ to avoid the rise time of the benzene excimer and intermolecular dynamics.

\section{Potential Energy Surface}

To rationalize the long excited state lifetime and the low quantum yield and chemoselectivity of the HFB $4 \pi$-electrocyclic isomerization, we computed potential energy surface along two independent degrees of freedom towards Dewar-HFB (Figure 7). The reaction coordinates are the out-of-plane angles of two carbon atoms that form the new carbon-carbon $\sigma$ bond in Dewar-HFB. All other degrees of freedom are allowed to relax for each point on the PES, and the constrained geometries were optimized with SA-10-CASSCF(6,7)/aug-cc-pVDZ. The resulting PES in the ground and ${ }^{1} B_{2 u}$ excited states is shown in Figure 7, along with the geometries of several critical points in the reaction pathway. 


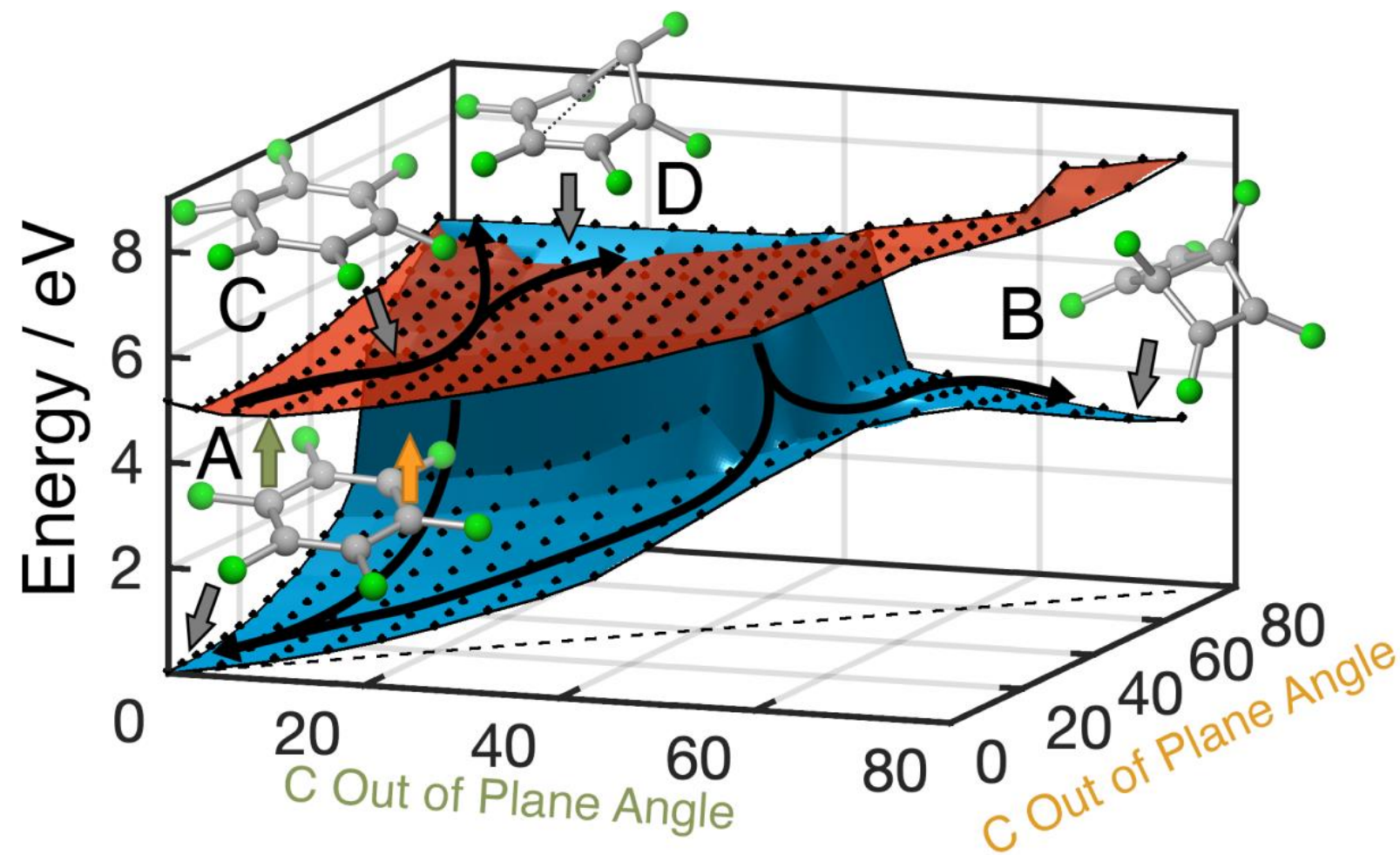

Figure 7. A $2 \mathrm{D}$ cut through the $\mathrm{S}_{0}{ }^{1} \mathrm{~A}_{1 \mathrm{~g}}$ (blue) and ${ }^{1} \mathrm{~B}_{2 \mathrm{u}}$ potential energy surfaces. The two coordinates correspond to the angle of the two para carbons, indicated by the orange and green arrows, rising out of the plane defined by the other four carbons. Motion diagonally across the PES from the $D_{6 H}$ minimum in the left-hand corner (A) to the right hand corner corresponds to both carbons rising symmetrically out of the plane to form the bridgehead bond and yield the DewarHFB isomer (B). Here both carbons are out of plane by $76^{\circ}$. Partial motion along the diagonal, where carbons are out of plane by 8 corresponds to the minimum energy geometry of the ${ }^{1} \mathrm{~B}_{2 \mathrm{u}}$ state (C). Motion along only one axis, where only one carbon rises out of plane, leads to the MECI at $65^{\circ}$ (D). From this MECI, internal conversion to the ground state leads exclusively to the D6H minimum (A). However, from the MECI motion of the other carbon is not degeneracy breaking until $40^{\circ}$. Molecules which pass through the $\mathrm{CI}$ at the upper portion of the seam find themselves on a section of the ground state which bifurcates, with one trajectory leading back to (A) while the other trajectory leads to the Dewar form (B).

The PES shows that both the $\mathrm{S}_{0}$ and $\mathrm{S}_{1}$ surfaces feature a broad, energetic minimum centered at $0^{\circ}$ and $8^{\circ}$, respectively, though the $\mathrm{S}_{1}$ surface is significantly flatter. The wide range of available motion in this flat region around the $\mathrm{S}_{1}$ minimum likely accounts for the fast 360 fs dampening of the oscillations in the transient absorption data. The surfaces also show that there is an $\mathrm{S}_{1} / \mathrm{S}_{0}$ crossing seam, which lies $\geq 1.4 \mathrm{eV}$ above the $\mathrm{S}_{1}$ minimum geometry (structure $\mathrm{C}$ ). This seam corresponds to one out-of-plane angle of $>65^{\circ}$, and the $\mathrm{S}_{1} / \mathrm{S}_{0}$ degeneracy persists for geometries where the opposing out-of-plane angle is $<40^{\circ}$. The geometry of the minimum-energy crossing 
point (MECP) on this crossing seam is shown as structure D. The two out-of-plane angles in the MECP geometry are $76^{\circ}$ and $0^{\circ}$, analogous to the $\mathrm{S}_{1} / \mathrm{S}_{0} \mathrm{MECP}$ in benzene. ${ }^{28}$

Non-adiabatic relaxation from the $\mathrm{S}_{1}$ state requires at least $1.4 \mathrm{eV}$ more energy than the $\mathrm{S}_{1}$ minimum, suggesting that this process would proceed slowly-if at all. This is in line with the TA measurements that indicate a long excited-state lifetime. Time Correlated Single Photon Counting (TCSPC) measurements, shown in Figure S7 and Table S5, confirm this lifetime approaches 2.29 ns. The ground-state surface near the crossing seam also shows that relaxation via geometries near the MECP results in a steep path back to the HFB reactant rather than Dewar-HFB. Thus, the crossing seam's productive region lies at higher energies, where the crossing geometry more closely resembles the photoproduct. This region of the seam is up to $0.3 \mathrm{eV}$ higher than the MECP, indicating this process is less favorable than relaxation back to the reactant explaining the low quantum yield for the photoisomerization. It is not however immediately evident from the potential energy surface why hexafluoro-benzvalene is not an observed HFB photoproduct after passing through the MECP in HFB as it is in the analogous benzene.

The branching spaces which define the MECPs in HFB and benzene are analyzed using the firstorder approximation of Galván et al. ${ }^{32}$ The reaction pathway to benzvalene from the MECP lies along a vector in the branching space, labeled $\hat{\mathbf{g}}$. The relative slope of the PES along $+\hat{\mathbf{g}}$ and $-\hat{\mathbf{g}}$ is shown in Figure 8, along with the MECP geometry and atom-wise contributions to the $\hat{\mathbf{g}}$ vector. The ground state reaction pathway that leads away from the MECP towards $-\hat{\mathbf{g}}$ leads to a reformation of the $D_{6 h}$ minimum benzene geometry, while $+\hat{\mathbf{g}}$ leads to the formation of benzvalene.
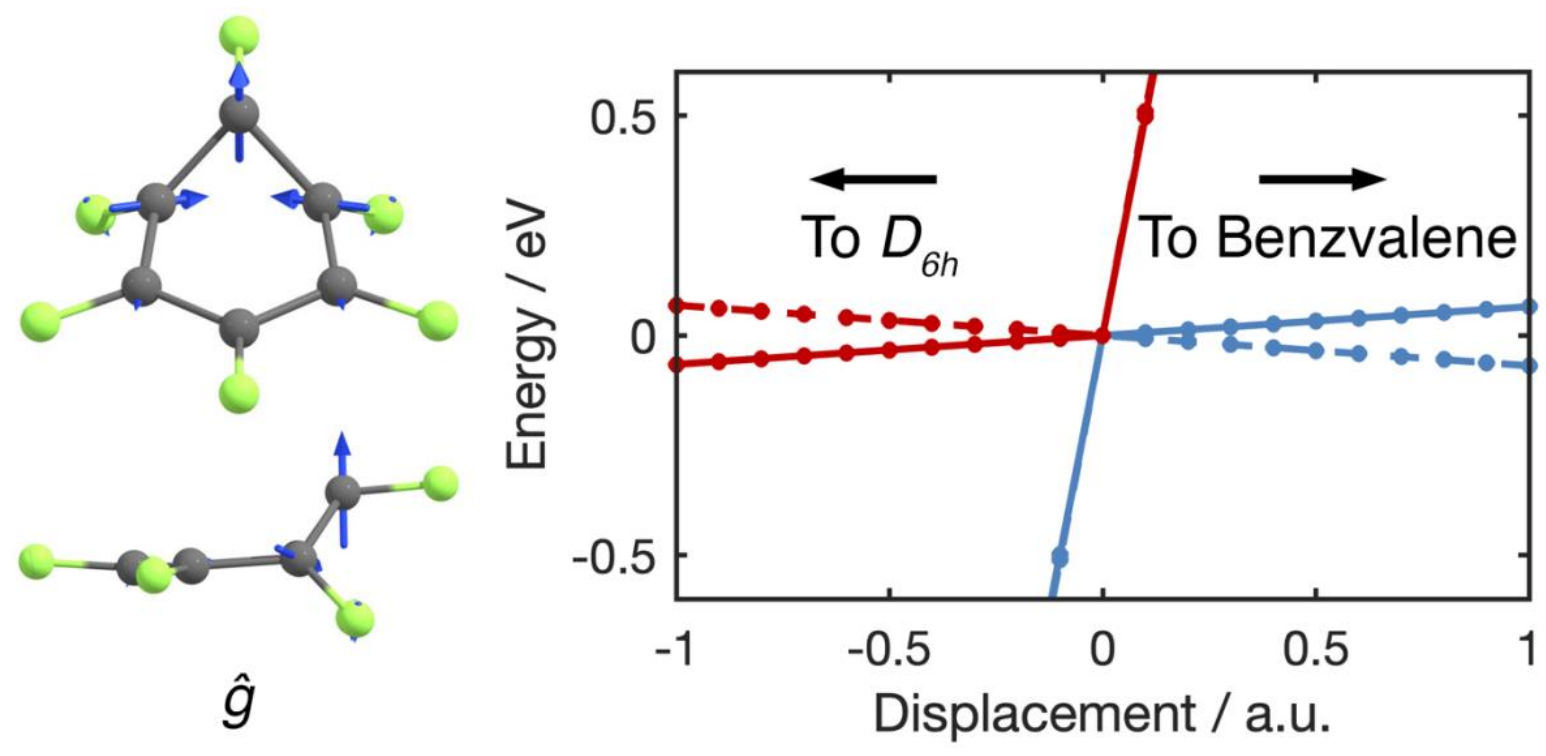

Figure 8. (Left) Minimum Energy CI geometry in HFB with atom-wise contributions to the $\hat{\mathbf{g}}$ vector. (Right) Cuts along the branching space $\hat{\mathbf{g}}$ vector for HFB (solid) and benzene (dashed).

In benzene's branching space, shown by the dashed lines in Figure 8, the excited state surface slopes downwards towards the MECP from both $+\hat{\mathbf{g}}$ and $-\hat{\mathbf{g}}$ directions, forming an energetic funnel. The ground state surface shows that relaxation away from the MECP along both $+\hat{\mathbf{g}}$ and $-\hat{\mathbf{g}}$ directions is also downhill, though $-\hat{\mathbf{g}}$ is overwhelmingly preferred. These surfaces suggest that benzene molecules nonadiabatically relaxing through this MECP will primarily reform the reactant benzene, with a small benzvalene yield. This analysis agrees well with the observed benzvalene 
isomerization quantum yield of $1 \% .{ }^{11}$ The branching space of HFB is shifted compared to benzene, such that both of the interacting surfaces increase in energy from $-\hat{\mathbf{g}}$ to $+\hat{\mathbf{g}}$. This subtle difference has profound effects on the photochemistry through this branching space. The excited-state surface no longer forms a funnel. Instead, a downhill pathway exists in the excited state along $-\hat{\mathbf{g}}$. The ground state surface of HFB is sloped away from $+\hat{\mathbf{g}}$, which further disfavors the formation of hexafluoro-benzvalene. The shift in the PES slope is a product of the PJT coupling between the ${ }^{1} B_{2 u}$ and ${ }^{1} E_{1 g}$ states, which lowers the energy of the ${ }^{1} B_{2 u}$ minimum, thereby increasing the curvature of the PES. Thus, while benzvalene is an observed photochemical product for benzene, that reaction pathway is destabilized in HFB, effectively shutting down this reaction channel and enforcing the observed chemoselectivity in the (overall inefficient) photoisomerization.

\section{Simulated Photochemical Dynamics}

Static calculations of the PES provide important mechanistic insight but lack information on the dynamic molecular evolution or the relative rates of competing excited-state processes. To understand the time-resolved excited state structural dynamics of HFB, an ensemble of gas phase non-adiabatic dynamics trajectories are computed using the surface-hopping including arbitrary coupling method as implemented in the SHARC suite of programs. ${ }^{33-35}$ Initial conditions for these trajectories were sampled from the Wigner distribution and initialized in the $S_{1}$ and $S_{2}$ electronic states. Each trajectory was propagated for $1 \mathrm{ps}$ with a 0.5 fs time step. We propagated 100 trajectories from the $S_{1}$ state and 400 trajectories from the $S_{2}$ state.

For the $\mathrm{S}_{1}$ trajectories, only three reach the ground state in the simulation time, making a statistical analysis of the rate constant unreliable. However, both of those trajectories reform the HFB reactant upon reaching the ground state. These observations are again in line with the experimentally observed long-lived excited state and low reaction quantum yield.

Although we have not carried out the corresponding experiment, considering the behaviour of HFB molecules excited to the $\mathrm{S} 2\left({ }^{1} \mathrm{E}_{1 \mathrm{~g}}\right)$ is informative. The time evolution of the state populations for the ensemble of $S_{2}$ trajectories is shown in the top panel in Figure 9. $397(99.25 \%)$ of these trajectories nonadiabatically relaxed to the $\mathrm{S}_{1}$ state, and $189(47.25 \%)$ went on to reach the ground state within the $1 \mathrm{ps}$ simulation window. This is due to the additional kinetic energy provided by initial excitation to a higher-energy electronic state. Kinetic fitting of these populations yields two time constants. The $S_{2}-S_{1}$ relaxation process occurs with a time constant of $129 \pm 5 \mathrm{fs}$, and the $\mathrm{S}_{1-}$ $\mathrm{S}_{0}$ relaxation fits to a time constant of $1180 \pm 90 \mathrm{fs}$. The initial $\mathrm{S}_{2}-\mathrm{S}_{1}$ relaxation agrees well with the previously determined values $0.10-0.54$ ps based on femtosecond time-resolved ion yield measurements for gas phase HFB. ${ }^{19}$

Trajectories deposited on the $S_{1}$ surface at the $S_{2} / S_{1}$ conical intersection are deposited near the $S_{1}$ minimum but with more kinetic energy than those prepared in the FC region and consequently relax faster than the $2.29 \mathrm{~ns}$ time constant observed in TCSPC following excitation to $\mathrm{S}_{1}$. This result is consistent with the decreasing $\mathrm{S}_{1}$ fluorescence quantum yield observed in Figure $\mathrm{S}$. 

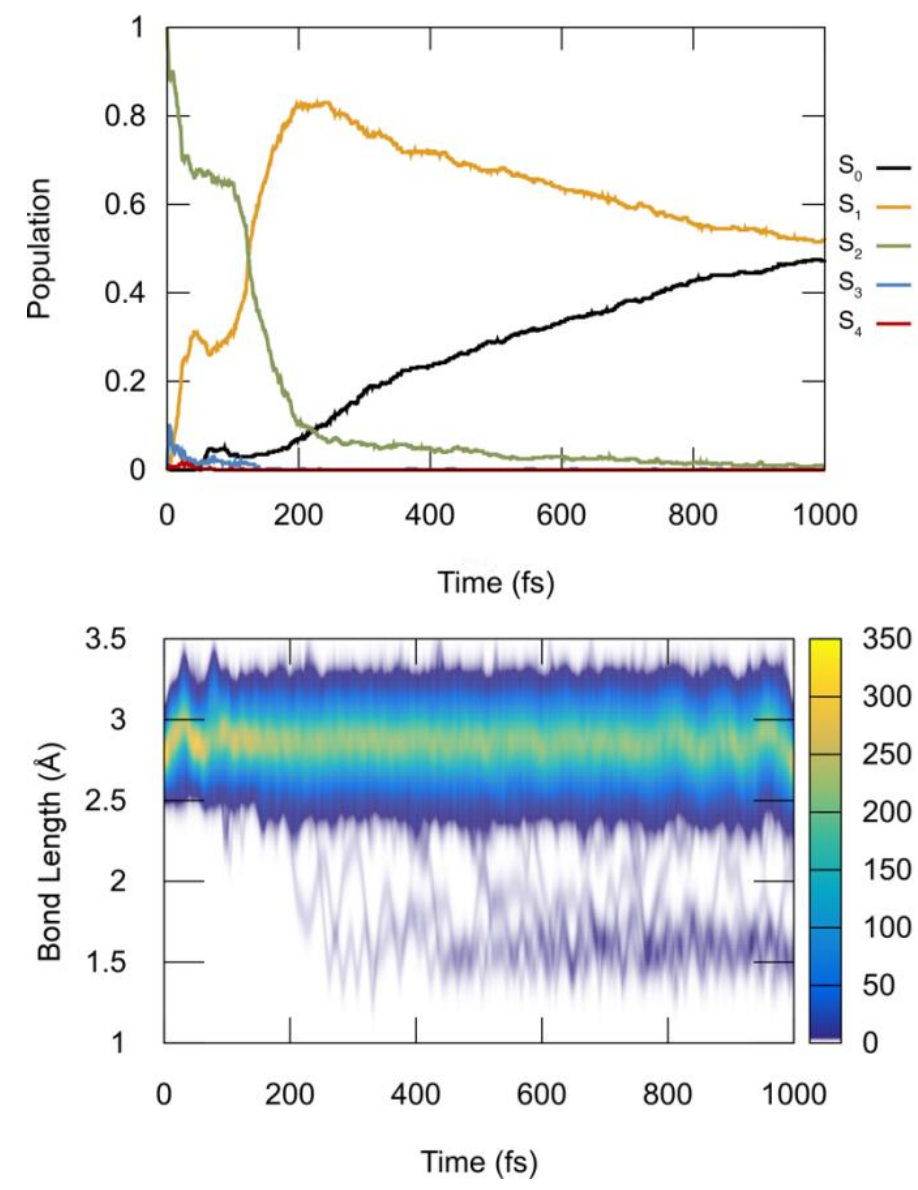

Figure 9. (Left) Time evolution of the population of each electronic state. Green trace shows $\mathrm{S}_{2}$, orange trace shows $\mathrm{S}_{1}$, and black trace shows $\mathrm{S}_{0}$. (Right) Time evolution of reactive $\mathrm{C}-\mathrm{C}$ bond distance for full ensemble of 400 trajectories.

The bottom of Figure 9 shows the geometric evolution of the 400 trajectories initialized on $\mathrm{S}_{2}$ throughout the simulation time. Of the 189 trajectories which reach the ground state surface in the simulation time, $96.8 \%$ (183) of these follow a reaction channel that reverts to the HFB reactant, similar to the $\mathrm{S}_{1}$-initialized trajectories. However, the remaining 6 trajectories follow a different reaction channel, which does not reform HFB and instead forms the experimentally observed photoproduct, Dewar-HFB. These trajectories appear in the figure at a reactive bond length of $<2$ $\AA$. While statistical analysis of such a small number of trajectories is unreliable, these data do show that the isomerization reaction can occur directly from non-adiabatic relaxation to the ground state through higher-energy geometries in the $\mathrm{S}_{1} / \mathrm{S}_{0}$ crossing seam.

Further, none of the photochemically productive trajectories pass through a ground state biradical intermediate, as was proposed by Varma et al. Instead, the softening of the ${ }^{1} B_{2 u}$ surface along the $e_{2 u}$ vibrational modes promotes molecular vibrations that resemble the Dewar-HFB photoproduct while simultaneously rendering the competing photochemical pathway unfavorable. This is sufficient to produce a small yield of Dewar-HFB from an ensemble of photoexcited HFB molecules, on the order of $1 \%$.

It would appear from our analysis that under the conditions typically used in synthesis $(254 \mathrm{~nm}$, $80 \mathrm{~W}, 8$ hours), the rather small fraction of molecules born on $\mathrm{S}_{2}$ may be responsible for the scant 
photoproduct formed. Why this photochemical preparation works at all under synthesis conditions would seem to come down to the product accumulating slowly over long irradiation times due to the kinetic stability of Dewar-HFB and its lack of absorption in the near-UV region.

It is thus clear that the strength of hexafluorobenzene derivatives as synthetic reagents, in contrast to hydrogenated analogs, is not just the presence of a pathway towards Dewar-HFB on $\mathrm{S}_{1}$ but a strict chemoselective photoproduct formation. In the demonstration of this reaction by Burns et al., the ratio of photons to molecular reagents was $168: 1$ and achieved a $41 \%$ synthetic yield. ${ }^{4}$ Interpreting this through the lens of these results shows that although most absorption events result in the reformation of the $D_{6 h} \mathrm{HFB}$, provided there is a small but exclusive pathway leading to Dewar-HFB, constant upcycling of the HFB by using a high molar excess of photons still yields an effective reaction.

\section{Conclusions}

HFB and its derivatives are photochemically reactive moieties employed synthetically for their photochemical chemoselective isomerization to Dewar-HFB and its analogs. Ultrafast transient absorption spectroscopy, multireference calculations, and non-adiabatic molecular dynamics simulations have been employed to understand the chemoselectivity, which makes this highly inefficient reaction still synthetically useful. Experimental and simulated absorption spectra show that the perfluoro effect is responsible for lowering the energy of the ${ }^{l} E_{1 g}\left(\pi \sigma^{*}\right)$ state to $\mathrm{S}_{2}, 0.42$ $\mathrm{eV}$ above the ${ }^{1} B_{2 u}\left(\pi \pi^{*}\right)$ state. As a result, these states are then vibronically couple along $e_{2 u}$ vibrational modes, distorting the excited state minimum geometry towards the Dewar-HFB product. This perturbation of the potential energy surface creates a barrier towards hexafluorobenzvalene, which enforces the Dewar-HFB chemoselectivity despite it still being a disfavored channel when compared with reformation of the $D_{6} \mathrm{~h}$ reactant. These results demonstrate that the internal $4 \pi$ electrocyclic ring closing is promoted by the electronegative perfluoro effect. Molecular dynamics simulations of photoexcitation to higher electronic states suggest that this would further increase the reaction rate towards Dewar-HFB formation, but this would require experimental confirmation to determine the magnitude of yield enhancement. This work serves as a foundation for understanding the photochemistry of highly fluorinated systems, paving the way for the targeted synthesis of high-value strained materials.

\section{Acknowledgements}

J.C. and S.A.L. acknowledge Dr. Jingbai Li for helpful discussion on the NAMD trajectories. S.A.L. and S.B. acknowledge the Office of Naval Research (ONR N00014-12-1-0828) for funding this research. J.C. and S.A.L. appreciate the assistance from the Northeastern Research Computing Team and access to the computing resources of the Discovery cluster. 


\section{Methods and Materials}

\section{Computational Methods}

The complete active space self-consistent field (CAS) method has been used to model the photochemical isomerization of hexafluorobenzene with a 6 electron, 7 orbital active space shown in Figure 10. The lowest-energy $\mathrm{C}-\mathrm{F} \sigma^{*}$ orbital is in the active space as previous experimental and computational studies on hexafluorobenzene show that this orbital is energetically stabilized to a level at or below the lowest $\pi^{*}$ orbital due to the perfluoro effect. This $\sigma^{*}$ orbital can participate in some of the lowest-lying excited states, and therefore must be included in the active space for an accurate description of, at least, the vertical excitation region of the PES. To account for dynamic electron correlation, CAS energies were corrected with second-order perturbation theory, including electronic state interactions with extended multi-state formulation (XMS-CASPT2) ${ }^{36}$
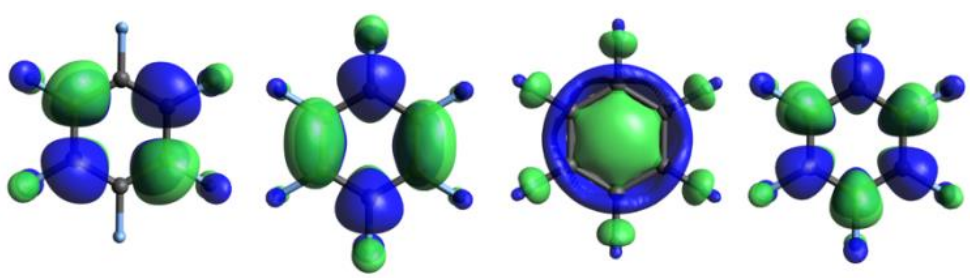

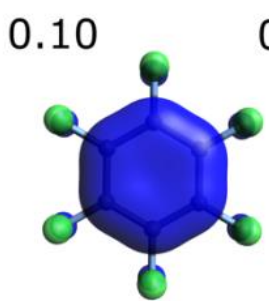

1.96
0.10

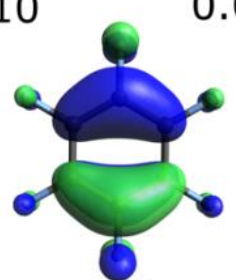

1.90
0.00

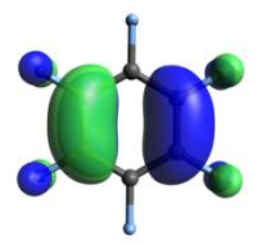

1.90

Figure 10. Orbitals included in the CASSCF active space and their average occupation in the ground state wavefunction. Orbitals are computed at the SA-10-CAS(6,7)/aug-cc-pVDZ level.

\section{Absorption and Emission Spectra}

The vibrationally resolved absorption and emission spectra were simulated by computing the overlap between the ground- and excited-state vibrational wavefunctions (Franck-Condon factors) using the harmonic approximation, as implemented in ezSpectrum. ${ }^{26}$ The intensities of vibrational bands were computed for combination bands of 13 vibrational normal modes with 30 vibrational quanta distributed among them. The geometries, normal modes, and vibrational frequencies were taken from the optimized minimum geometries on the corresponding PES at the CAS(6,7)/aug-ccPVDZ level.

The steady-state absorption spectrum was simulated by averaging the absorption spectrum of 150 geometries taken from a single ground-state ab initio molecular dynamics trajectory $(300 \mathrm{~K})$. The trajectory was first equilibrated for $50 \mathrm{fs}$ at $300 \mathrm{~K}$, followed by a $450 \mathrm{fs}$ production simulation. The snapshots used in the absorption spectrum were randomly sampled from this trajectory, and the absorption energies and oscillator strengths were computed at the XMS-CASPT2(6,7)/aug-ccPVDZ level. Each absorption peak in each stick spectrum was convoluted with a gaussian function with a full-width half-max of $0.24 \mathrm{eV}$. 


\section{Non-Adiabatic Molecular Dynamics}

Photochemical reaction dynamics were simulated using the surface hopping, including an arbitrary coupling algorithm implemented in the SHARC suite of programs. Initial conditions for each trajectory were sampled from the Wigner distribution in the ground state. An ensemble of 100 trajectories was initialized in the $\mathrm{S}_{1}$ and $\mathrm{S}_{2}$ electronic states; an additional 300 trajectories were initialized in the $\mathrm{S}_{2}$ state. Trajectories propagated for 1 picosecond with the NVE ensemble and a time step of 0.5 femtoseconds. The electronic structure at each time step was computed at the SA5-CAS $(6,6)^{\prime} /$ ANO-S-VDZP level. The $(6,6)^{\prime}$ active space used here eliminates the highest-energy $\pi^{*}$ orbital from the $(6,7)$ space described above. This was previously reported to improve the ordering of electronic states in conjugated systems when a CASPT2 correction is not performed. ${ }^{31}$ $\operatorname{CASSCF}(6,7)$ predicts that the $\mathrm{S}_{2}$ state has doubly-excited $\left(\pi^{*} \pi^{*}\right)$ character, which is corrected via XMS-CASPT2 to the level of S7. This intruder state is absent from the frontier excited states in CASSCF $(6,6)^{\prime}$. The dynamics simulations employ the ANO-S-VDZP basis set; it achieves comparable accuracy to the aug-cc-pVDZ basis at a computational cost comparable to cc-pVDZ. ${ }^{37}$

\section{Sample Preparation and Purification}

Hexafluorobenzene (Sigma Aldrich, $>99 \%$ purity) was used without further purification for the transient absorption and TCSPC measurements. For fluorescence and UV-Vis experiments, HFB was purified by fractional distillation at $120^{\circ} \mathrm{C}$ to remove fluorescent impurities (Figure S8). All HFB solutions were made with ethanol (VWR, 200 proof).

Distillation was performed using a fractional distillation column and a water jacketed condenser. The HFB was boiled in a round-bottom flask using mineral oil to a bath temperature of $120{ }^{\circ} \mathrm{C}$. The first few drops of the distillate, the head, was removed from the collection flask and discarded. The first distillate was then collected and redistilled again. The HFB used in long-wavelength UV-vis experiments, both distilled and non-distilled, was then diluted via serial dilution and used for the experiment.

Tryptophan (Sigma Aldrich, 97\%) was used as the fluorescence quantum yield standard without further purification.

\section{Absorption and Fluorescence Measurements}

All absorption measurements were performed on a Cary $60 \mathrm{UV} / \mathrm{VIS}$ spectrometer with ethanol in a quartz $1 \mathrm{~cm}$ cuvette used to set the baseline. The fluorescence measurements were carried out on a Horiba Jobin Yvon FluoroMax-3 fluorometer. For fluorescence measurements, the sample was loaded into a $1 \mathrm{~cm}$ quartz cuvette, and the bandwidths of both the excitation and emission monochromators were set to $2 \mathrm{~nm}$ and scanned in $0.25 \mathrm{~nm}$ increments with each point integrated for $100 \mathrm{~ms}$. All spectra were background-subtracted against pure solvent captured under the same conditions.

In the quantum yield measurements, the absorbances of the HFB and tryptophan/buffer solutions at $270 \mathrm{~nm}$ excitation were $0.034 \mathrm{OD}$ and $0.049 \mathrm{OD}$, respectively. A buffer solution without tryptophan produced no fluorescence at $270 \mathrm{~nm}$ excitation.

The wavelength-dependent fluorescence quantum yield (Figure S10) was calculated by normalizing the HFB absorption and fluorescence excitation at $270 \mathrm{~nm}$, dividing this by the absorption and scaling by 0.029 - the fluorescence quantum yield of tryptophan at $270 \mathrm{~nm}$. 


\section{Temperature-Dependent Fluorescence}

Temperature-dependent fluorescence was recorded using a QuantaMaster Xe arc lamp spectrofluorometer equipped with an R1527 PMT detector loaded with a $71 \mu \mathrm{M}$ solution of HFB in ethanol in a $1 \mathrm{~cm}$ screw-top quartz Starna Cells cell. The excitation monochromator was centered at $255 \mathrm{~nm}$, while the emission monochromator was stepped in $1 \mathrm{~nm}$ intervals from 300 to $500 \mathrm{~nm}$. The integration time was set to $1 \mathrm{~s}$, and the excitation and emission monochromator bandwidth slits were set to $5 \mathrm{~nm}$. Scans were averaged twice. The sample cell is immersed in a temperature-controlled flowing water bath which, was varied between 23 and $56{ }^{\circ} \mathrm{C}$.

\section{Femtosecond Transient Absorption}

The $35 \mathrm{fs}, 800 \mathrm{~nm}$ output of a Coherent Legend amplifier was taken to generate both the pump and probe beams. $10 \%$ was sent to generate the white light continuum probe. The time delay between the pump and probe was generated by sending the probe down a translating gold-plated cube corner retroreflector. The white light was generated by focusing the fundamental onto a $2 \mathrm{~mm}$ rotating $\mathrm{CaF}_{2}$ disk. The supercontinuum was collimated and then focused on the sample by two off-axis parabolic mirrors. The transmitted probe beam was collimated and then focused onto a $120 \mu \mathrm{m}$ slit of a grating Czerny-Turner monochromator. The light was then dispersed across a 256-pixel array to achieve a detected continuum spanning from $345 \mathrm{~nm}$ to $650 \mathrm{~nm}$.

The deep UV (DUV) pump beam was generated in a home built non-colinear optical parametric amplifier (NOPA) pumped with $60 \mathrm{~mW}$ of $800 \mathrm{~nm}(35 \mathrm{fs})$. About $10 \%$ of the fundamental was focused on a sapphire disk to generate a white light seed. The remaining fundamental was directed and focused on an SHG BBO to generate the $400 \mathrm{~nm}$ pump. The signal was generated by combining the weak white light seed onto a thin BBO crystal with the $400 \mathrm{~nm}$ pump. The signal generated, $510 \mathrm{~nm}$, is then passed through a prism compressor to compensate for spectral chirp of the visible beam and increase DUV SHG efficiency. The signal is then sent through another BBO crystal to generate the second harmonic of $510 \mathrm{~nm}, 255 \mathrm{~nm}$. The DUV beam was sent through two calcium fluoride prisms to compress the UV pulses. The DUV pulse was focused to a spot size of $360 \mu \mathrm{m}$ with a pulse energy of $580 \mathrm{~nJ}, 5 \mathrm{~cm}$, in front of the gravity jet sample. ${ }^{38}$ A gravity jet was used in place of a sample flow cell to reduce nonlinear effects such as cross-phase modulation and possible signal from the glass and reduce temporal walk off of the pump and probe pulses due to spectral chirp, optimizing the time resolution. For this setup, the sample was contained within an upper reservoir that flowed downward, forming a thin sheet across a loop of 0.005 " tungsten wire, forming a jet with a thickness of $130 \mu \mathrm{m}$ at the pump-probe overlap. The pump and probe were overlapped at the gravity jet sheet in the center of the loop to minimize lensing effects. The reservoir height was maintained at a constant height of $\sim 40 \mathrm{~cm}$ to ensure a consistent sheet thickness throughout the experiment.

To compensate for the low signal to noise ratio, the spectra were averaged in delay time using a 10 point moving mean algorithm. This improves the signal-to-noise ratio from 16 to 87 when assessed at the same $300 \mathrm{fs}, 550 \mathrm{~nm}$ point.

\section{Time-Correlated Single Photon Counting}

The fluorescence lifetime of HFB was measured using Time-Correlated Single Photon Counting (TCSPC). $532 \mathrm{~nm}$, produced as the $2^{\text {nd }}$ harmonic of an Alphalas PULSELAS-A-266-300-SP Nd: YAG laser operating at $10 \mathrm{kHz}$, was focused into a fourth harmonic generation (FHG) crystal to 
yield $266 \mathrm{~nm}$, which was used to pump the sample. The sync trigger is provided by the residual $532 \mathrm{~nm}$ passing through the $266 \mathrm{~nm}$ pick off the high reflector. This residue was used to trigger a Becker \& Hickl GmbH PHD-400-N fast photodiode sync.

The experiment was done in a $1 \mathrm{~cm}$ quartz cuvette with a sample absorption of $0.043 \mathrm{OD}$ at 266 $\mathrm{nm}$. The total time acquisition of the TCSPC measurements was 60 minutes with an intensity of $150 \mathrm{~mW} / \mathrm{cm}^{2}$. The power was set such that the number of detected photons was lower than $\sim 1 \%$ of the laser repetition rate. This was to reduce the probability of two detected events per laser shot, which can distort the timed-binned histogram. 


\section{Bibliography}

(1) Watson, W. H.; McMordie, W. C.; Lands, L. G. Polymerization of Alkynes by Ziegler-type Catalyst. J Polym Sci 1961, 55 (161), 137-144. https://doi.org/10.1002/pol.1961.1205516114.

(2) Shirakawa, H.; Louis, E. J.; MacDiarmid, A. G.; Chiang, C. K.; Heeger, A. J. Synthesis of Electrically Conducting Organic Polymers: Halogen Derivatives of Polyacetylene, $(\mathrm{CH})$ x. $J$ Chem Soc Chem Commun 1977, o (16), 578-580. https://doi.org/10.1039/c39770000578.

(3) Gould, G. L.; Eswara, V.; Trifu, R. M.; Castner, D. G. Polydifluoroacetylene, Polychlorofluoroacetylene, and Polydichloroacetylene. J Am Chem Soc 1999, 121 (15), 37813782. https://doi.org/10.1021/ja983840a.

(4) Boswell, B. R.; Mansson, C. M. F.; Cox, J. M.; Jin, Z.; Romaniuk, J. A. H.; Lindquist, K. P.; Cegelski, L.; Xia, Y.; Lopez, S. A.; Burns, N. Z. Mechanochemical Synthesis of an Elusive Fluorinated Polyacetylene. Nat Chem 2021, 13 (1), 41-46. https://doi.org/10.1038/s41557-02000608-8.

(5) Ward, H. R.; Wishnok, J. S. Vacuum Ultraviolet Photolysis of Liquid Benzene. Photoisomerization of Benzene to Dewar Benzene. J Am Chem Soc 1968, 90 (4), 1085-1086. https://doi.org/10.1021/ja01006a058.

(6) Bryce-Smith, D.; Gilbert, A.; Orger, B. H. Photoaddition of Cis -Cyclo-Octene to Hexafluorobenzene. J Chem Soc D Chem Commun 1969, 0 (14), 800b-8802. https://doi.org/10.1039/c2969000800b.

(7) Zupan, M.; Šket, B. Photochemistry of Fluorosubstituted Aromatic and Heteroaromatic Molecules. Israel J Chem 1978, 17 (1-2), 92-99. https://doi.org/10.1002/ijch.197800012.

(8) Šket, B.; Zupančič, N.; Zupan, M. Photochemistry of Organo-Halogenic Molecules. Part 20. The Effect of Cycloalkene Structure on the [2+2] Photocycloaddition to Hexafluorobenzene. $J$ Chem Soc Perkin Transactions 1 1987, O (0), 981-985. https://doi.org/10.1039/p19870000981.

(9) Lemal, D. M. Hexafluorobenzene Photochemistry: Wellspring of Fluorocarbon Structures. Accounts Chem Res 2001, 34 (8), 662-671. https://doi.org/10.1021/ar960057j.

(10) Haller, I. Photoisomerization of Hexafluorobenzene. J Am Chem Soc 1966, 88 (9), 20702071. https://doi.org/10.1021/ja00961a055.

(11) Wilzbach, K. E.; Ritscher, J. S.; Kaplan, L. Benzvalene, the Tricyclic Valence Isomer of Benzene. J Am Chem Soc 1967, 89 (4), 1031-1032. https://doi.org/10.1021/ja00980a053.

(12) Blair, J. M.; Bryce-Smith, D. The Photoisomerization of Benzene to Fulvene. Proc Chem Soc 1957, O (October), 287-288. https://doi.org/10.1039/ps9570000273. 
(13) Zgierski, M. Z.; Fujiwara, T.; Lim, E. C. Photophysics of Aromatic Molecules with LowLying П $\sigma^{*}$ States: Fluorinated Benzenes. J Chem Phys 2005, 122 (14), 144312. https://doi.org/10.1063/1.1873752.

(14) Clark, I. D.; Frost, D. C. A Study of the Energy Levels in Benzene and Some Fluorobenzenes by Photoelectron Spectroscopy. J Am Chem Soc 1967, 89 (2), 244-247. https://doi.org/10.1021/ja00978a011.

(15) Camaggi, G.; Gozzo, F.; Cevidalli, G. Para -Bonded Isomers of Fluoroaromatic Compounds. Chem Commun Lond 1966, 0 (10), 313-314. https://doi.org/10.1039/c19660000313.

(16) Haller, I. Kinetics and Mechanism of the Photochemical Valence Tautomerization of Hexafluorobenzene. J Chem Phys 1967, 47 (3), 1117-1125. https://doi.org/10.1063/1.1711996.

(17) L., S., J.; H., H., A.; O., V., C. A. G. Picosecond Spectroscopy in Study of the Photoinduced Isomerization of Hexafluorobenzene. Laser Chem 1986, 6 (5), 333-347. https://doi.org/10.1155/lc.6.333.

(18) Cordes, T.; Herzog, T. T.; Malkmus, S.; Draxler, S.; Brust, T.; DiGirolamo, J. A.; Lees, W. J.; Braun, M. Wavelength and Solvent Independent Photochemistry: The Electrocyclic RingClosure of Indolylfulgides. Photochem Photobiol Sci 2009, 8 (4), 528-534. https://doi.org/10.1039/b817627b.

(19) Studzinski, H.; Zhang, S.; Wang, Y.; Temps, F. Ultrafast Nonradiative Dynamics in Electronically Excited Hexafluorobenzene by Femtosecond Time-Resolved Mass Spectrometry. J Chem Phys 2008, 128 (16), 164314. https://doi.org/10.1063/1.2907859.

(20) Motch, C.; Giuliani, A.; Delwiche, J.; Limão-Vieira, P.; Mason, N. J.; Hoffmann, S. V.; Hubin-Franskin, M.-J. Electronic Structure of Hexafluorobenzene by High-Resolution Vacuum Ultraviolet Photo-Absorption and He(I) Photoelectron Spectroscopy. Chem Phys 2006, 328 (13), 183-189. https://doi.org/10.1016/j.chemphys.2006.05.032.

(21) Holland, D. M. P.; Shaw, D. A.; Stener, M.; Decleva, P. A Study of the Valence Shell Electronic Structure of Hexafluorobenzene Using Photoabsorption and Photoelectron Spectroscopy, and TDDFT Calculations. J Phys B Atomic Mol Opt Phys 2009, 42 (24), 245201. https://doi.org/10.1088/0953-4075/42/24/245201.

(22) Philis, J.; Bolovinos, A.; Andritsopoulos, G.; Pantos, E.; Tsekeris, P. A Comparison of the Absorption Spectra of the Fluorobenzenes and Benzene in the Region 4.5-9.5 EV. J Phys B Atomic Mol Phys 1999, 14 (19), 3621. https://doi.org/10.1088/0022-3700/14/19/013.

(23) Mondal, T.; Reddy, S. R.; Mahapatra, S. Photophysics of Fluorinated Benzene. III. Hexafluorobenzene. J Chem Phys 2012, 137 (5), 054311. https://doi.org/10.1063/1.4739502. 
(24) Taniguchi, M.; Lindsey, J. S. Database of Absorption and Fluorescence Spectra of $>300$ Common Compounds for Use in PhotochemCAD. Photochem Photobiol 2018, 94 (2), 290-327. https://doi.org/10.1111/php.12860.

(25) Berlman, I. B. Handbook of Fluorescence Spectra of Aromatic Molecules; 1971. https://doi.org/10.1016/b978-0-12-092656-5.x5001-1.

(26) Mozhayskiy, V. A.; Krylov, A. I. EzSpectrum; 2021.

(27) Goodman, L.; Ozkabak, A. G.; Thakur, S. N. A Benchmark Vibrational Potential Surface: Ground-State Benzene. J Phys Chem 1991, 95 (23), 9044-9058.

https://doi.org/10.1021/j100176a008.

(28) Palmer, I. J.; Ragazos, I. N.; Bernardi, F.; Olivucci, M.; Robb, M. A. An MC-SCF Study of the S1 and S2 Photochemical Reactions of Benzene. J Am Chem Soc 1993, 115 (2), 673-682. https://doi.org/10.1021/ja00055a042.

(29) Nakashima, N.; Sumitani, M.; Ohmine, I.; Yoshihara, K. Nanosecond Laser Photolysis of the Benzene Monomer and Eximer. J Chem Phys 1980, 72 (4), 2226-2230.

https://doi.org/10.1063/1.439465.

(30) Miyazaki, M.; Fujii, M. Real Time Observation of the Excimer Formation Dynamics of a Gas Phase Benzene Dimer by Picosecond Pump-Probe Spectroscopy. Phys Chem Chem Phys 2015, 17 (39), 25989-25997. https://doi.org/10.1039/c5cp03010b.

(31) Thompson, A. L.; Martínez, T. J. Time-Resolved Photoelectron Spectroscopy from First Principles: Excited State Dynamics of Benzene. Faraday Discuss 2011, 150 (0), 293-311. https://doi.org/10.1039/c1fd00003a.

(32) Galván, I. Fdez.; Delcey, M. G.; Pedersen, T. B.; Aquilante, F.; Lindh, R. Analytical StateAverage Complete-Active-Space Self-Consistent Field Nonadiabatic Coupling Vectors: Implementation with Density-Fitted Two-Electron Integrals and Application to Conical Intersections. J Chem Theory Comput 2016, 12 (8), 3636-3653.

https://doi.org/10.1021/acs.jctc.6b00384.

(33) Mai, S.; Richter, M.; Hendl, M.; Menger, M. F. S. J.; Atkins, A.; Ruckenbauer, M.; Plasser, F.; Ibele, L. M.; Kropf, S.; Oppel, M.; Marquetand, P.; González, L. SHARC2.1: Surface Hopping Including Arbitrary Couplings - Program Package for Non-Adiabatic Dynamics; 2021.

(34) Richter, M.; Marquetand, P.; González-Vázquez, J.; Sola, I.; González, L. SHARC: Ab Initio Molecular Dynamics with Surface Hopping in the Adiabatic Representation Including Arbitrary Couplings. J Chem Theory Comput 2011, 7 (5), 1253-1258. https://doi.org/10.1021/ct1007394.

(35) Mai, S.; Marquetand, P.; González, L. Nonadiabatic Dynamics: The SHARC Approach. Wiley Interdiscip Rev Comput Mol Sci 2018, 8 (6), e1370. https://doi.org/10.1002/wcms.1370. 
(36) Shiozaki, T.; Győrffy, W.; Celani, P.; Werner, H.-J. Communication: Extended Multi-State Complete Active Space Second-Order Perturbation Theory: Energy and Nuclear Gradients. $J$ Chem Phys 2011, 135 (8), 081106. https://doi.org/10.1063/1.3633329.

(37) Plasser, F.; Mewes, S. A.; Dreuw, A.; González, L. Detailed Wave Function Analysis for Multireference Methods: Implementation in the Molcas Program Package and Applications to Tetracene. J Chem Theory Comput 2017, 13 (11), 5343-5353.

https://doi.org/10.1021/acs.jctc.7b00718.

(38) Tauber, M. J.; Mathies, R. A.; Chen, X.; Bradforth, S. E. Flowing Liquid Sample Jet for Resonance Raman and Ultrafast Optical Spectroscopy. Rev Sci Instrum 2003, 74 (11), 49584960. https://doi.org/10.1063/1.1614874.

\section{For Table of Contents Only}

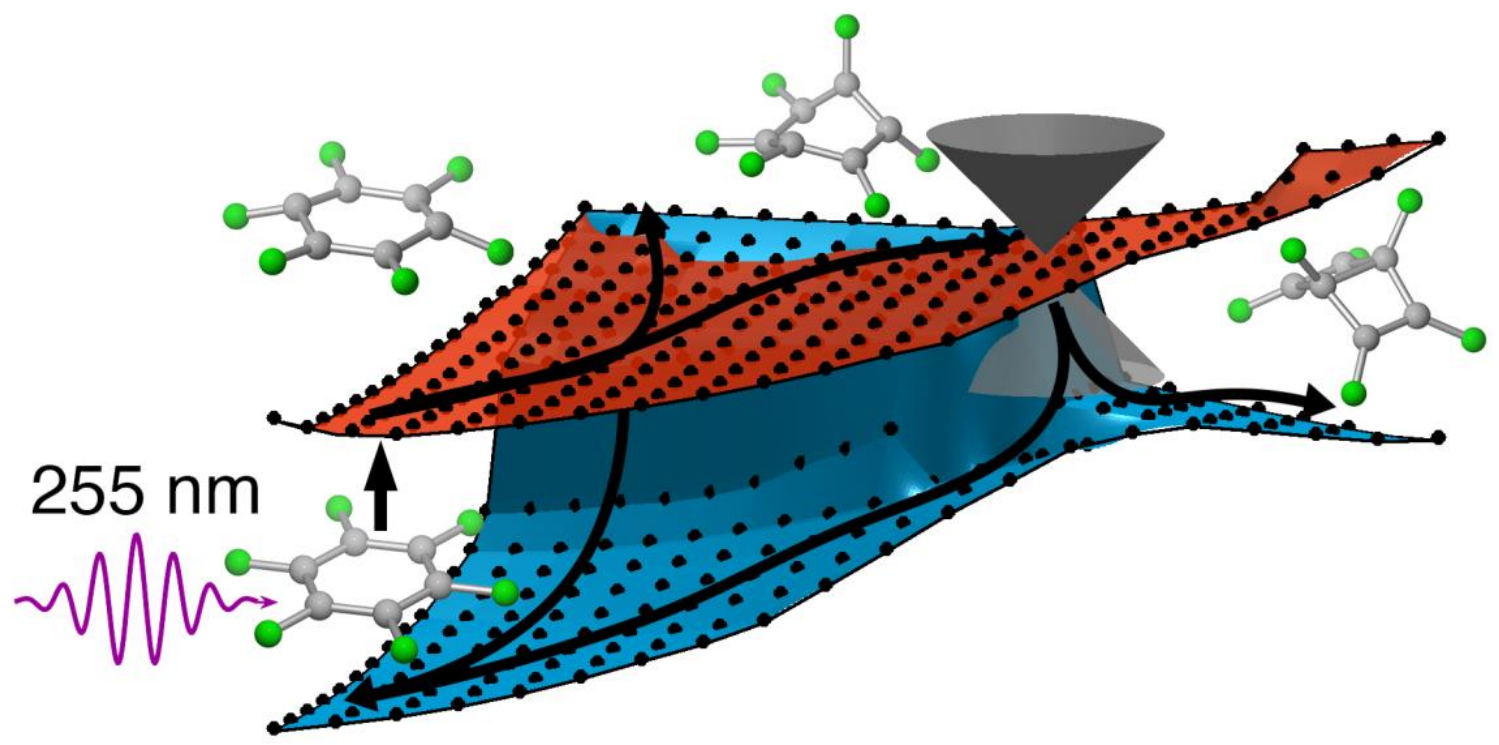

